\title{
Kalibrierung von Thermometern in situ im Prozess
}

\author{
$\frac{\text { Marc Schalles }}{}{ }^{1}$, \\ ${ }^{1}$ Institut für Prozessmess- und Sensortechnik der Technischen Universität IImenau, PSF 100565, \\ 98684 IImenau, Deutschland \\ ${ }^{2}$ Endress+Hauser Wetzer GmbH + Co.KG, Obere Wank 1, 87484 Nesselwang,
}

\begin{abstract}
Zusammenfassung
Im Bereich der Life-Sciences-Technology müssen Prozessthermometer aufgrund regulatorischer Anforderungen regelmäßig kalibriert werden. Derzeit müssen diese aus dem Prozess ausgebaut und extern kalibriert werden, was, bedingt durch die Kalibrierung oder auch durch Prozessstillstände, sowohl zu hohen Aufwänden, als auch zu hohen Kosten führt.

Um diese Kosten zu minimieren und die Prozesssicherheit zu erhöhen, wurde ein Kalibrierverfahren entwickelt, welches eine kontinuierliche Einpunktkalibrierung eines Thermometers direkt im Prozess ermöglicht. Diese in-situ-Kalibrierung erfolgt dabei mit Hilfe eines in das Thermometer integrierten Referenzmaterials, dessen Curie-Temperatur $T_{\mathrm{C}}=118^{\circ} \mathrm{C}$ die Referenztemperatur der Kalibrierung bereitstellt. Die Kalibrierung findet damit nahe der für Dampfsterilisationen nachzuweisenden Temperatur von $121^{\circ} \mathrm{C}$ statt, sodass die Richtigkeit der Temperaturmessung gerade bei dieser kritischen Temperatur kontinuierlich automatisch überprüft werden kann. Die Unsicherheit der in-situKalibrierung beträgt $U= \pm 349 \mathrm{mK}$.
\end{abstract}

Keywords: In-situ-Kalibrierung,Inline,Steam in Place,Cleaning in Place,SIP-CIP

\section{Einleitung}

Die häufigste im Bereich der Prozessmessung erfasste und für den Herstellungsprozess relevante Größe ist die Temperatur. An ihre richtige Messung bestehen insbesondere in der pharmazeutischen oder Lebensmittelindustrie hohe Anforderungen. Aufgrund der existierenden betrieblichen und behördlichen Vorschriften ist eine regelmäßige Kalibrierung der Messstellen obligatorisch. Diese Kalibrierung dient beispielsweise der Erhöhung der Prozesssicherheit und -effizienz und auch der Minimierung von Ausfallzeiten.

Bei der Kalibrierung, die typischerweise halbjährlich oder jährlich durchgeführt wird, werden die Thermometer aus dem Prozess ausgebaut und in einer externen Kalibriereinrichtung kalibriert. Dabei entsteht aufgrund der Montage- und Kalibrierdauer sowohl ein hoher zeitlicher, als auch ein hoher finanzieller Aufwand, da sich beispielsweise in großen Prozessanlagen durchaus mehrere tausend Thermometer befinden können. Trotz des hohen Aufwands, kann häufig bei diesen Kalibrierungen weder bei prozesstypischen Bedingungen, noch die gesamte Messkette kalibriert werden, sodass die prozesstypischen Messabweichungen nicht exakt abgebildet werden können. Außerdem liegen im Zeitraum zwischen den Kalibrierungen keine Informationen zur Messgenauigkeit der Thermometer vor.

Wäre eine automatische Kalibrierung von Thermometern direkt im Prozess möglich, könnte die gesamte Messkette vom Sensor bis zum Transmitter unter Prozessbedingungen überprüft werden. Zusätzliche ergäbe sich ein sehr hohes Einsparpotential bei den Kalibrierkosten und eine kontinuierliche Überwachung der Messstelle.

Ein Thermometer, welches erstmalig eine solche automatische Kalibrierung ermöglicht, wurde in einer Forschungskooperation der Firma Endress+Hauser und dem Institut für Prozessmess- und Sensortechnik der TU Ilmenau entwickelt. Seine Kalibrierung basiert auf einem dynamischen Kalibrierverfahren, bei welchem die Curie-Temperatur eines in das Thermometer integrierten Referenzmaterials, eine physikalisch feste und bekannte Bezugstemperatur der Kalibrierung darstellt. Die Kalibrierung des Thermometers wird beim Erreichen der Curie-Temperatur automatisch durch die Prozesstemperatur ausgelöst und die Abweichung des mit der gesamten Thermometermesskette bestimmten digitalen Temperaturwerts bestimmt.

\section{Stand der Technik der In-situ-Kalibrierung}

Zur Kalibrierung von Thermometern, muss generell eine für die angestrebte Unsicherheit der Kalibrierung ausreichend genau bekannte Referenztemperatur $T_{\text {ref }}$ bereitgestellt werden. Mit dieser wird die Temperaturanzeige des zu kalibrierenden Thermometers, des Prüflings $T_{\mathrm{s}}$, verglichen. Hierfür können zwei verschiedene Kalibrierprinzipien, die Fixpunktkalibrierung und die Vergleichskalibrierung, verwendet 
werden. Beide werden bei Kalibrierungen außerhalb von Prozessen, beispielsweise in Kalibrierlaboratorien genutzt.

Bei der Fixpunktkalibrierung dient die bekannte Temperatur einer Phasenumwandlung eines definierten Materials als Referenztemperatur. Ein Beispiel hierfür ist die Phasenumwandlung vom flüssigen in den festen Zustand von Reinstwasser, welche unter Atmosphärendruck (Normbedingung) bei $T_{\text {ref }}=0{ }^{\circ} \mathrm{C}$ stattfindet. Realisiert man diese Phasenumwandlung in einer geeigneten Einrichtung, wie einem EisWasser-Gemisch in einem Dewargefäß, so kann der Prüfling in das Wasser eingetaucht und sein Sensor idealerweise auf die Temperatur der Phasenumwandlung $\left(T_{\mathrm{s}}=T_{\text {ref }}\right)$ gebracht werden. Die Abweichung der gemessenen Temperatur $T_{\mathrm{M}}$ vom Referenzwert $\Delta T_{\mathrm{M}}=T_{\mathrm{M}}-T_{\text {ref }}$ oder eines Ausgabewertes $X_{\mathrm{M}}\left(T_{\mathrm{M}}\right)$ ist das Ergebnis der Kalibrierung

Eine alternative und in der Praxis relevantere Methode der Kalibrierung stellt die Vergleichskalibrierung dar. Hier wird beispielsweise mit einem Ofen oder einem Kalibrierbad eine Umgebung möglichst homogener Temperatur bereitgestellt, in der sich der Prüfling, sowie ein Referenzthermometer befinden. Idealerweise werden auch hier beide Thermometer auf die gleiche Temperatur $T_{\mathrm{s}}=T_{\text {ref, }}$ gebracht. Die Temperatur $T_{\text {ref }}$ der Kalibrierumgebung wird mittels des genau kalibrierten Referenzthermometers bestimmt und zu diesem die Abweichung $\Delta T_{\mathrm{M}}$ ermittelt.

Beide Methoden, die aus der LaborKalibrierung bekannt sind, werden auch aktuell bei In-situ-Kalibrierungen im Prozess verwendet.

\section{In-situ-Fixpunktkalibrierungen}

In-situ-Fixpunktkalibrierungen finden vorwiegend in Prozessen bei hohen Temperaturen, beispielsweise in Kraftwerken, und mit Thermoelementen als Temperatursensoren statt. Als Fixpunktmaterialien werden hier eutektische Metall-Kohlenstoff- oder MetallMetallkarbidlegierungen [1] aber auch Reinstmetalle und Metall-Metallegierungen [2, 3] verwendet. Die prinzipielle Bauform solcher Miniaturisierten Fixpunktzellen ist in Abb. 1 dargestellt.

Eine mit dem Fixpunktmaterial gefülte Zelle ist in der Spitze eines Thermometermesseinsatzes untergebracht. In die Zelle selbst ist der Sensor des Prüflings, hier ein Thermoelement, eingesteckt.Er kann so die Prozesstemperatur, als auch bei Stattfinden einer Phasenumwandlung des Fixpunktmaterials die sich im Inneren der Zelle einstellende Phasenumwandlungstemperatur messen. Der
Messeinsatz selbst befindet sich dabei im Prozess. Die Phasenumwandlung und damit der Kalibriervorgang kann beim Überschreiten der Phasenumwandlungstemperatur durch die Prozesstemperatur ausgelöst werden. Alternativ kann dies bei sehr stabilen Prozesstemperaturen auch mittels einer in den Messeinsatz integrierten Heizung geschehen.
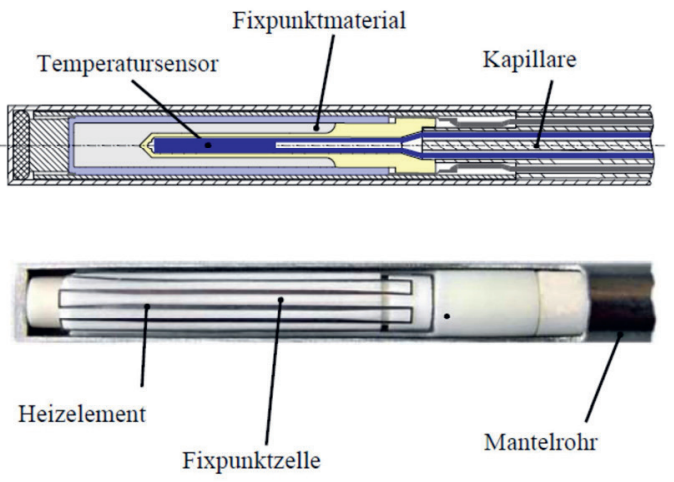

Abb. 1: Schnitt eines Messeinsatzes eines Thermometers mit Miniaturisierter Fixpunktzelle [4]

Der Vorteil der In-situ-Kalibrierung mittels Miniaturisierten Fixpunktzellen besteht in der direkten Rückführbarkeit der Kalibrierung auf eine physikalisch bekannte Temperatur. Nachteilig bei dieser Art der Kalibrierung ist, dass das Thermometer aufgrund der während der Phasenumwandlung umgesetzten Latenten Wärme vom Prozess entkoppelt ist und die Prozesstemperatur in diesem Bereich selbst nicht erfasst werden kann. Außerdem ist die Bauform der Thermometer sehr komplex.

\section{In-situ-Vergleichskalibrierung}

Bei der In-situ-Vergleichskalibrierung wird die Temperatur der Kalibrierung durch den Prozess vorgegeben und ist variabel. Neben dem eigentlichen Prozesssensor des Prüflings ist mindestens ein weiterer sog. Referenzsensor, in den Messeinsatz integriert. Diese Referenzsensoren sind in der Regel Thermoelemente, Widerstandssensoren oder Kombinationen aus beiden $[5,6]$. Da hier sowohl Prüfling, als auch Referenzsensoren den Prozessbedingungen ausgesetzt sind, unterliegen alle einer Alterung. Um trotz der Alterung der Referenzsensoren eine möglichst sichere Messung der Referenztemperatur zu ermöglichen, wird versucht die alterungsbedingte Kennliniendrift der Referenz zu kompensieren. Dies geschieht durch Verwendung verschiedener Sensorarten mit unterschiedlicher oder bekannter Kennliniendrift und einer mathematischen oder elektrischen Korrektur. Dieser Ansatz ist jedoch wenig erfolgreich, da die Alterung bzw. Kennliniendrift von Pt-Widerstandssensoren, 
die in der Life-Sciences-Industrie häufig eingesetzt werden, selbst schon sehr gering ist. Ebenso können Veränderungen an der Messelektronik zusätzlich eine unerkannte Verfälschung der Referenztemperaturbestimmung hervorrufen.

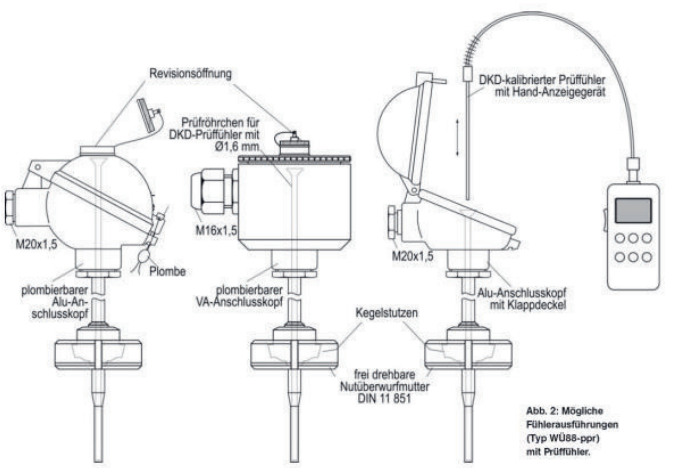

Abb. 2: Technische

Ansichten des prozessprüfbaren Thermometersystems [7]

Bei sogenannten prozessprüfbaren Thermometersystemen (Abb. 2) wird die Alterung des Referenzthermometers dadurch minimiert, dass selbiges nicht im Prozess verbleibt. Es wird vielmehr für jeden Kalibriervorgang manuell von extern in den im Prozess befindlichen Messeinsatz mit Prüfling gesteckt. Bei der Kalibrierung wird dann die Anzeige beider Geräte verglichen. Der Nachteil bei dieser Art von Inline-Kalibrierung besteht darin, dass bei jedem Kalibriervorgang eine manuelle Einrichtung des Referenzthermometers an der Messstelle nötig ist, wodurch ein signifikanter personeller und finanzieller Aufwand für die Kalibrierung bleibt. Auch muss das Referenzthermometer selbst regelmäßig rückführbar kalibriert werden.

\section{In-situ-Kalibrierung am Curie-Punkt}

Ein neuartiges Verfahren zur In-situKalibrierung stellt die dynamische Kalibrierung am Curie-Punkt dar, welches im Rahmen einer Forschungskooperation der TU IImenau und der Firma Endress+Hauser entwickelt wurde. Die Ergebnisse dieser Kooperation wurden im Produkt iTHERM TrustSens umgesetzt, welches die Firma Endress+Hauser vertreibt. Dieses Verfahren nutzt erstmalig die CurieTemperatur $T_{\mathrm{C}}$ eines polarisierten Materials als Referenztemperatur $T_{\text {ref. }}$ Die Curie-Temperatur ist die Temperatur, bei deren Überschreiten ein polarisiertes Material seinen polarisierten Zustand verliert. Wie die Schmelz- oder Tripelpunkttemperatur auch, ist die CurieTemperatur eine materialspezifische Eigenschaft, die in einem Material bestimmter Reinheit, Zusammensetzung oder Struktur reversibel existiert. An der Curie-Temperatur verbleibt das Material sowohl im polarisierten, als auch im unpolarisierten Zustand in der festen Phase. Der Polarisationszustand kann mittels verschiedener elektrischer Messverfahren detektiert und somit das Vorliegen der Phasenumwandlung erkannt werden.

Die Grundlage des Verfahrens bildet somit eine reproduzierbare sowie langzeitstabile Phasenumwandlung, deren Auftreten sicher detektiert werden kann. Durch die Verwendung von Ferroelektrika als Referenzmaterial, beispielsweise $\mathrm{PbTiO}_{3}, \mathrm{~Pb}\left[\mathrm{Zr}_{\mathrm{x}} \mathrm{Ti}_{1-\mathrm{x}}\right] \mathrm{O}_{3}$ oder $\mathrm{SrTiO}_{3}$, ist dies sichergestellt. Bei diesen findet an der Phasenumwandlungstemperatur ein abrupter Polarisationswechsel statt, wie es in der folgenden Abbildung dargestellt ist. Der Polarisationswechsel, hier bei $T_{\mathrm{C}}=125^{\circ} \mathrm{C}$, erzeugt eine Änderung der Dielektrizitätskonstante um mehrere hundert Prozent, die im elektrischen Signal der Auswertung eindeutig erfasst werden kann.

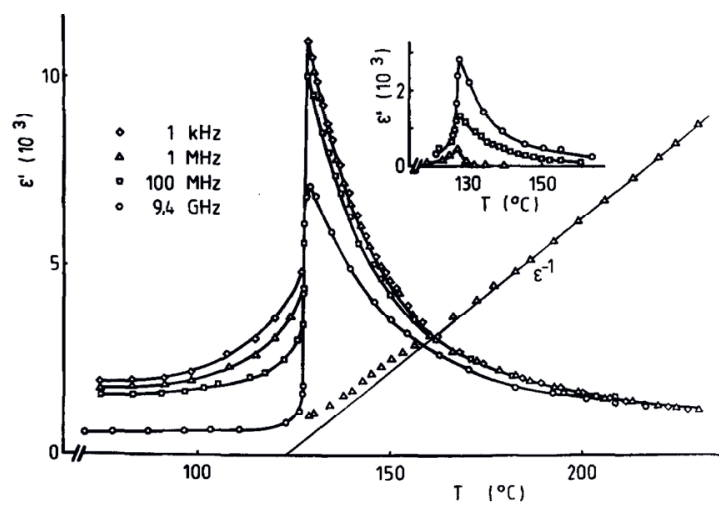

Abb. 3: Dispersion des Realteils $\varepsilon^{\prime}$ und des Imaginärteils $\varepsilon^{\prime \prime}$ der Dielektrizitätskonstante einer $\mathrm{BaTiO}_{3}$-Probe mit $\mathrm{T}_{\mathrm{C}}=125^{\circ} \mathrm{C}[8]$

Im Gegensatz zu den meist bei stationären Kalibierverfahren des Stands der Technik, bei denen zur Kalibrierung eine hinreichend konstante Temperatur $T_{\text {ref }}$ und ein thermisches Gleichgewicht $T_{\text {ref }}=T_{\mathrm{s}}$ eingestellt wird, liegt bei dieser neuartigen Kalibriermethode ein dynamisches Kalibrierverfahren vor.

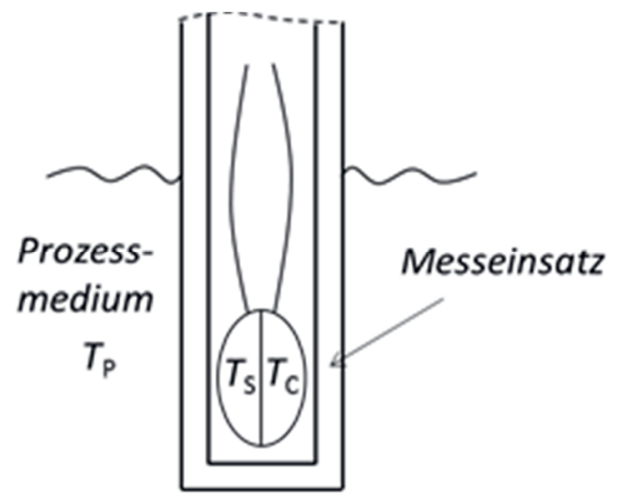

Abb. 4: Skizze zur Anordnung von Referenz mit $T_{C}$ und Prozesssensor mit $T_{s}$ in einem Messeinsatz 
Bei diesem wird durch das Prozessmedium, der Temperatur $T_{\mathrm{p}}$, eine Temperaturänderung im Messeinsatz erzeugt, die dabei das im Messeinsatz befindliche Referenzmaterial, sowie den Prozesssensor gleichermaßen erfasst. Durch die angepasste Konstruktion des spezifisch entwickelten Messeinsatzes ist dabei gewährleistet, dass Referenzmaterial und Prozesssensor immer die hinreichend gleiche Temperatur besitzen. Wird nun bei der Temperierung die bekannte Curie-Temperatur überschritten, wird dies durch die die Polarisation detektierende Elektronik erfasst und der Zeitpunkt dieser Phasenumwandlung festgestellt. Da zu diesem gleichzeitig auch der kontinuierlich messende Prozesssensor ausgelesen wird, kann ermittelt werden, ob eine Abweichung $\Delta T=T_{\mathrm{s}}-T_{\mathrm{C}}$ vorliegt.

\section{Messergebnisse}

Die Phasenumwandlungstemperatur beträgt beim neu entwickelten In-situ-Kalibriersystem $T_{\mathrm{C}}=118{ }^{\circ} \mathrm{C}$. Sie wurde speziell an SIP-CIPProzesse angepasst, bei denen mittels Heißdampf eine Reinigung und Sterilisation der Prozessanlagen vorgenommen werden muss. Da bei diesen Reinigungszyklen eine Sterilisationstemperatur von mindestens $121{ }^{\circ} \mathrm{C}$ erreicht wird, wird bei jedem vorgenommenen Reinigungszyklus $T_{\mathrm{C}}$ überschritten und damit eine Phasenumwandlung und folglich eine Kalibrierung ausgelöst. Weil solche
Reinigungszyklen, abhängig vom Prozess, täglich oder mehrmals in der Woche stattfinden, erhält man bei der In-situKalibrierung eine kontinuierliche Überwachung des Thermometers nahe der nachzuweisenden Sterilisationstemperatur. Die gesamte Messkette kann somit an der CurieTemperatur kontinuierlich unter realen Prozessbedingungen überwacht werden.

Die Messunsicherheit der Kalibrierung mit dem In-situ-Kalibriersystem wurde in einer Messunsicherheitsbetrachtung zu $U= \pm 349 \mathrm{mK}$, bei $k=2(95 \%)$ bestimmt. Sie berücksichtigt unter anderem Einflüsse des Kalibrierverfahrens, der elektrischen Messwerterfassung, als auch der prozessspezifischen Temperierung.

Zur Untersuchung der In-situ-Kalibrierfunktion wurden 20 Thermometer kontinuierlich im Temperaturintervall $+30^{\circ} \mathrm{C} \ldots+160{ }^{\circ} \mathrm{C}$ in einem Ölbad temperiert. Dabei wurden bei jedem Thermometer 200 In-situ-Kalibriervorgänge ausgelöst und bei jeder Kalibrierung die Abweichung des Prozessthermometers zur Curie-Temperatur aufgezeichnet. Die Ergebnisse sind in Abb. 6 dargestellt. Gut zu erkennen ist die hohe Reproduzierbarkeit der Kalibrierungen, bei denen der überwiegende Teil der Kalibrierergebnisse innerhalb eines Bandes der Breite von $200 \mathrm{mK}$ liegen. Die Messergebnisse liegen somit deutlich innerhalb des im Diagramm eingezeichneten Unsicherheitsintervalls von $U= \pm 349 \mathrm{mK}$.

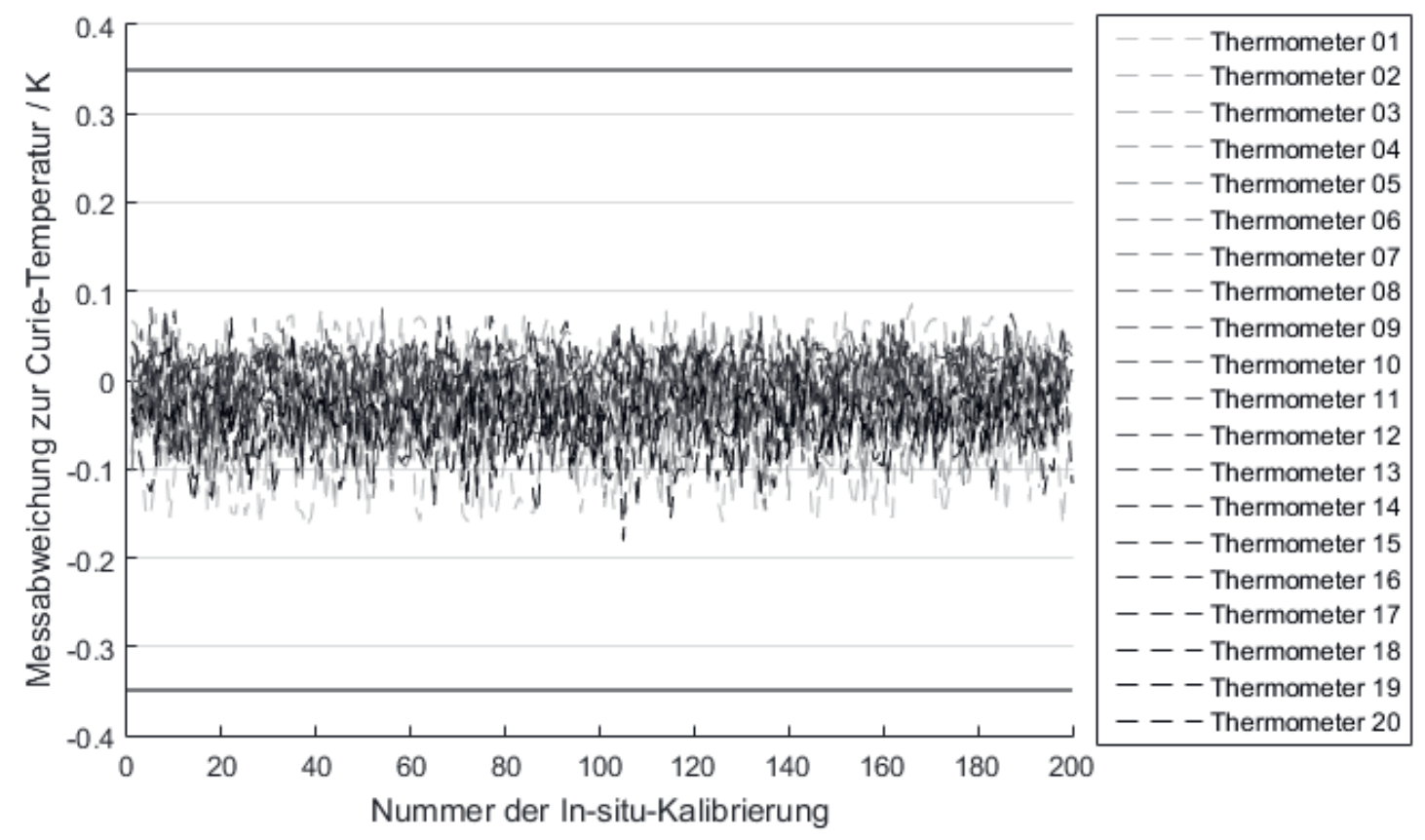

Abb. 6: In-situ-Kalibrierung von 20 Thermometern an der Curie-Temperatur und Ermittlung der Abweichung des Temperaturwerts zur nominellen Curie-Temperatur als Ergebnis der Kalibrierung. 
Um das Messsystem zusätzlich thermischen und mechanischen Belastungen auszusetzen, erfolgte während der Phase der Temperierung zusätzlich eine 7-tägige Alterung Messeinsätze bei der maximalen Einsatztemperatur von $+160{ }^{\circ} \mathrm{C}$ und 10 Temperaturschocks mit sprungförmigen Temperaturwechseln von $+160{ }^{\circ} \mathrm{C}$ auf $0{ }^{\circ} \mathrm{C}$ und zurück. Die Messungen zeigen, dass diese zusätzlichen Beanspruchungen keine Auswirkungen auf das Prozessthermometer mit dem Pt100 hatten. Die Kalibrierergebnisse änderten sich im Rahmen der Messunsicherheit nicht.

\section{Literaturnachweis}

[1] Elliott, C.J., et al., Metrologia 52(2), 267, (2015); doi:10.1088/0026-1394/52/2/267

[2] Krapf, G., et al, Temperatur 2006, Berlin: Physikalisch Technische Bundesanstalt, PTB (2006)

[3] Lehmann, H., International Journal of Thermophysics 31 (8-9), 1599-1607 (2010); doi:10.1007/s10765-010-0796-7

[4] Krapf, G., Schalles, M., Fröhlich, T., Measurement, 44(2), 385-390 (2010),

[5] Stone, P.W., Patent US4475823 (1984)

[6] Lunghofer, et al., Patent US5713668A, (1998)

[7] Kiesner, C., Matzen S., Deutsche Molkerei Zeitung 10 (2012.)

[8] Kersten, O., A. Rost, and G. Schmidt, Ferroelectrics 81, 31-34, (1988).

[9] https://www.de.endress.com/de/medien zentrum/news-pressemitteilungen/iTHERMTrustSens-selbstkalibrierendes-Thermometer 